\title{
Failed ventilation through the laryngeal mask airway
}

\author{
Takashi Asai
}

Received: 21 November 2013/Accepted: 29 November 2013/Published online: 20 December 2013

(C) Japanese Society of Anesthesiologists 2013

To the Editor:

The laryngeal mask airway (or other supraglottic airway) has a potential role in cases with a "cannot intubate, cannot ventilate" scenario [1], but it is important to elucidate possible causes of its failure [2] to establish the true role of the device in treating patients with difficult airways.

A 64-year-old man with brain edema was scheduled for brain lobectomy. One month ago, the patient had a traffic accident and underwent removal of subdural hemorrhage; facemask ventilation was possible, but tracheal intubation was accomplished with great difficulty. Preoperatively, his consciousness was impaired, and respiration followed a waxing and waning Cheyne-Stoke's-like pattern.

After induction of anesthesia and neuromuscular blockade, ventilation through a facemask was initially possible, but shortly after, it became impossible. Insertion of an intubating laryngeal mask did not allow ventilation. As the arterial hemoglobin oxygen saturation $\left(\mathrm{SpO}_{2}\right)$ remained $100 \%$, a fiberoptic bronchoscope and a tracheal tube were passed through the laryngeal mask to see if it was possible to see the glottis to achieve tracheal intubation. Fiberscopy revealed a sticky substance was totally obscuring the view of the glottis. All the devices were removed, and a half-dried thick sputum, $\sim 3 \times 3 \mathrm{~cm}$, was removed by suction. The laryngeal mask was reinserted, and adequate ventilation was obtained. Fiberoptic intubation through the laryngeal mask was successful.

Retrospectively, the waxing and waning respiration was likely caused by the sticky sputum. This report indicates that the laryngeal mask may fail if the glottis is obstructed by sticky sputum.

Conflict of interest None.

\section{References}

1. Asai T, Morris S. The laryngeal mask airway: its features, effects and role. Can J Anaesth. 1994;41:930-60.

2. Asai T. Strategies for difficult airway management-the current state is not ideal. J Anesth. 2013;27:1521-4.
T. Asai $(\bowtie)$

Department of Anesthesiology, Dokkyo Medical University,

Koshigaya Hospital, 2-1-50 Minamikoshigaya, Koshigaya,

Saitama 343-8555, Japan

e-mail: asaita@dokkyomed.ac.jp 